\title{
An Active Gain Control System of Avalanche Photo-Diodes for Phase-Shift Laser Range Finder
}

\author{
Jia Fang-xiu, YIN Ting-ting, ZHAI Meng, and WANG Xiao-ming \\ ZNDY of Ministerial Key Laboratory, Nanjing University of Science and Technology, Nanjing, \\ Jiangsu, 210094, China
}

Keywords: avalanche photodiode, multiplicatoin factor, control system, laser range finder.

\begin{abstract}
In time fight phase-shift laser range finder, it is noticed that unwanted phase delay in avalanche photo-diodes (APD) between the incident light and the output current. When the modulation frequency is higher and the incident light is weaker, the phase delay is becoming larger. The measurement resolution of laser range finder by the optical modulation method is strongly influenced by the undesirable phase delay. In order to solve the problem, the relationships among the phase delay, the reverse bias voltage and the multiplication factor of APD were investigated at different modulation frequency respectively. An active gain control system was proposed by controlling the reverse bias acting on the APD according to different incident light power. Given the high light intensity, the reverse bias voltage was adjusted to keep APD operating at optimal M factor, and the phase delay was compensated by software, on the condition that the low light intensity, the reverse bias voltage is decreased to reduce the phase delay. The experiment results show that for a modulation frequency of $75 \mathrm{MHz}$ and incident light intensity of $0.5 \mathrm{uW}$, the phase delay can be reduced from $4.8013^{\circ}$ to less than $0.04^{\circ}$.
\end{abstract}

\section{Introduction}

APD is a promising light sensor for various military fields such as laser range finder, laser radar, and laser identification of friend or foe because of its internal gain and high quantum efficiency, which can greatly reduce the performance requirement of pre-amplification circuits, and can improve the SNR of detecting circuits. Many researchers found that in case of pulse laser radar where the APD is exposed to wide dynamics of input pulses causing error in pulse time of flight measurement[1][2]. At the same time, in phase-shift laser range finder, with the increasing demand of accuracy in such applications, the modulation frequency is increasing higher up to hundreds' of $\mathrm{MHz}$; the nonlinearity of APD is becoming a hot research issue in phase-shift laser range finder[3][4]. Shuko Yokoyama and Akito Okamoto found that the degree of the phase delay increase with a decrease of the incident intensity, the distance measurement error will increase greatly when the incident light is weak; they concluded that the phase delay can be reduced by superimposing a bias light on the modulation light so that the total intensity remains constant[5]. Other researchers found that the phase delay is related to the light modulation frequency (or rising edge of the light pulse) and many correction systems were proposed in pulse laser range finder [6]-[10]. To clarify phase delay in phase-shift laser range finder, in our paper, a system has been designed, implemented and experimentally tested, the relationships among the phase delay, the reverse bias voltage acting on APD and the APD M factor. According to the experiment results, a system adjusting the reverse bias voltage flexibly was proposed that can not only suppress the phase delay but also ensures the SNR of the laser range finder.

\section{APD nonlinearity and phase delay measurement system}

\subsection{APD Nonlinearity}

The response of receive system in laser range finder is decided by the intrinsic response time of the $\mathrm{APD}$ and the RC time constant of the receive circuits. If the receive circuits is designed, the RC 
time constant is stable, and can be compensated. The intrinsic response time $\tau_{r}$ of the APD photoelectric signal is expressed by:

$$
\tau_{r}=\tau_{e}+\tau_{h}+\tau_{m}
$$

Where $\tau_{e}$ and $\tau_{h}$ is the transit time of electrons and holes through the absorption layer respectively, and $\tau_{m}$ is the multiplication time of the carriers. They can be expressed as:

$$
\begin{aligned}
\tau_{e} & =\frac{d_{a}}{v_{e}} \\
\tau_{h} & =\frac{d_{a}}{v_{h}} \\
\tau_{m} & =k M \frac{d_{g}}{v_{e}}+\frac{d_{g}}{v_{p}}
\end{aligned}
$$

Where $d_{a}$ is the absorption layer thickness, $v_{e}$ and $v_{h}$ are the saturation velocity of electrons and holes, $k$ is the ionization ratio and $d_{g}$ is the multiplication layer thickness of the APD. Even the multiplication time $\tau_{m}$ is lower than transit time $\tau_{e}+\tau_{h}$, but the phase delay is mainly due to the multiplication time [4]. If the structure of APD is set, and applied electric field is stable, $\tau_{m}$ increases with $\mathrm{M}$ factor, the relationship can be evaluated by:

$$
\delta t=k \cdot \delta M \cdot \frac{d_{g}}{v_{e}}
$$

So in phase-shift laser range finder, the phase-shift variation is mainly due to the $\mathrm{M}$ factor variation:

$$
\delta \varphi=2 \pi f_{k} k \cdot \delta M \cdot \frac{d_{g}}{v_{e}}
$$

Where, $f_{k}$ is the intensity modulation frequency in phase-shift laser range finder. In this case, the operation of APD as a receiver in laser range finder is shown in Fig. 1.

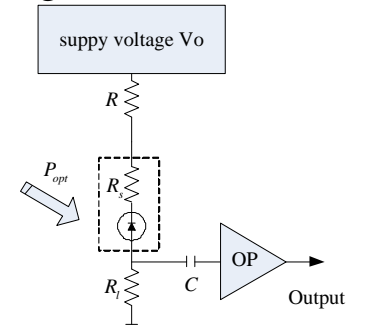

Fig. 1 Operation of APD as a receiver in laser range finder

The value of $\mathrm{M}$ factor is given by the inverse bias voltage $V_{H}$ acting on APD and breakdown voltage $V_{B}$ of the APD as:

$$
M(i)=\frac{1}{1-\left(\frac{V_{H}}{V_{B}}\right)^{n}}
$$

Where $\mathrm{n}$ is the concavity index of the avalanche zone and is 1.4-2.0 for silicon APD. According to Fig. 1:

$$
V_{H}=V_{O}-\left(R+R_{s}+R_{L}\right) i_{p h}
$$

Where $V_{o}$ is the supply voltage output, $R$ is the current-limiting resistance, $R_{s}$ is the APD inner resistance, $R_{l}$ is the load resistance, $i_{p h}$ is the average photocurrent, which decreases with the decreases of incident light power.

From equation (7) and (8), we can deduce that $V_{H}$ increases with incident light power decreasing, and the $\mathrm{M}$ factor increases. Combine with equation (6), the increase in phase delay can be explained by the decrease in the incident light intensity. And if the modulation frequency $f_{k}$ is higher, the phase delay will be larger which leads to the distance measurement error increase. 


\subsection{Phase delay measurement system}

From A section, we can conclude that the phase delay increase with the increasing of the modulation frequency and decreasing of the incident light power. To observe the phase delay by the nonlinearity of APD, the following system is designed in Fig. 2. The bias voltage is produced by PA92 and controlled by DSP through DAC, supplying APD1 and APD2 with variable high voltage at the same time (APD, Silicon Sensor, AD230-8, -3dB cutoff frequency 2GHz ). A laser diode (LD) is driven by a sinusoidal current at modulation of $75 \mathrm{MHz}$ and $7.5 \mathrm{MHz}$.

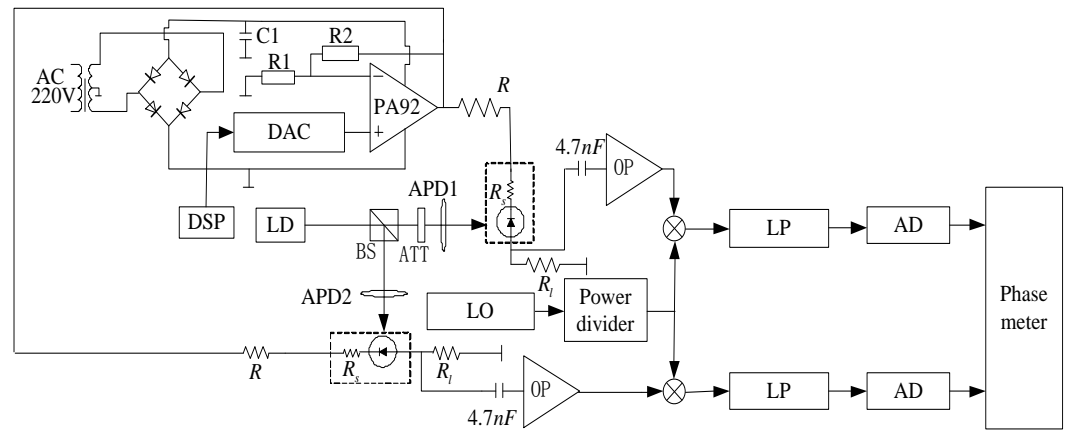

Fig. 2 Diagram of measuring phase error caused by nonlinear characterization of APD

Light from LD was divided into a reference beam illuminating the APD2 at fixed intensity of 50 $\mu \mathrm{W}$ and a measurement beam on APD1which intensity is adjustable between $0.5 \mu \mathrm{W} \sim 50 \mu \mathrm{W}$ by ATT. Local Oscillator (LO) signal after the power divider is mixed with measurement signal and the reference signal in convenient of the phase shift measurement. Two signals are sampled by ADC to phase meter employing apFFT method. The phase delays are shown in at modulation frequency of $75 \mathrm{MHz}$ and $7.5 \mathrm{MHz}$ when the bias voltage is set between $50 \mathrm{~V} \sim 147 \mathrm{~V}$.

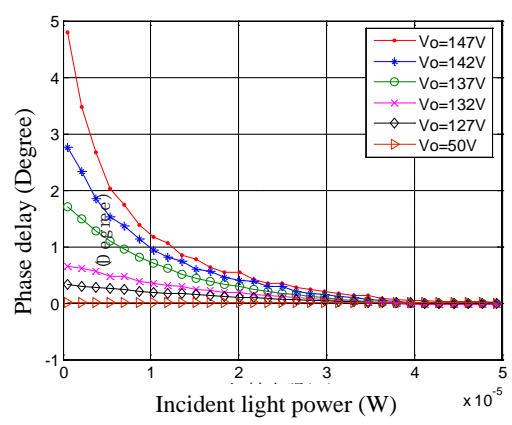

(a) Modulation frequency at $75 \mathrm{MHz}$

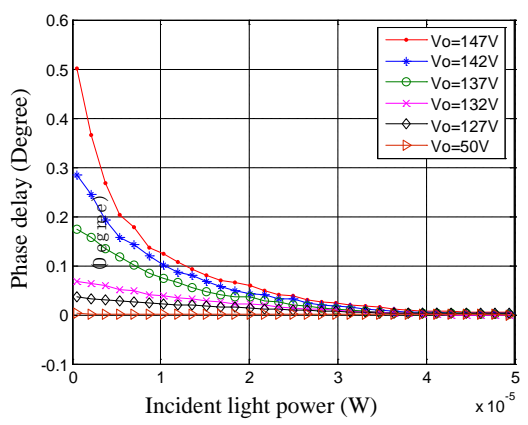

(b) Modulation frequency at $7.5 \mathrm{MHz}$

Fig. 3. Relation of incident light intensity and phase error

The maxim value of phase delay is $4.8013^{\circ}$ and $0.5018^{\circ}$ at modulation frequency of $75 \mathrm{MHz}$ and $7.5 \mathrm{MHz}$ respectively. The phase delay is increasing with the decreasing of light intensity, and at fixed light intensity, the phase delay is decreasing with the decrease of bias voltage $V_{o}$.when the bias voltage is set at $50 \mathrm{~V}$, the phase delay is near zero. It has been proven that APD is a single pole system, the cutoff frequency is $f_{c}$, so:

$$
f_{c} M=k(\text { : constant })
$$

For a phase-shift laser range finder at modulation frequency $f_{k}$, the phase delay can be written as:

$$
\Delta \varphi_{d}=\arctan \frac{f_{k}}{f_{c}}=\arctan \frac{f_{c} M}{k}
$$

According to equation (6) and (10), the phase delay is decided by the $\mathrm{M}$ factor. The Fig. 4 shows plot of the incident light intensity and multiplication factor $M_{\text {act }}$ for $V_{o}=127 \mathrm{~V} \sim 147 \mathrm{~V}$, the multiplication factor is unity when $V_{O}=50 \mathrm{~V}$.

$$
M_{a c t}=\frac{I_{p h} / v_{o}=V_{s}}{I_{p h} / V_{o}=50 V}
$$

Where $I_{p h}$ is the average photocurrent.

The $M_{\text {act }} \doteq 20$ with the increasing of incident light intensity. The $\mathrm{M}$ factor is increasing with the decrease of incident light power at fixed bias voltage. And reduce the bias voltage can make the $\mathrm{M}$ 
factor become a constant, which make the phase delay compensation become easy. So we can suppress the phase delay by adjusting the bias voltage, and if the bias voltage cannot be adjusted flexibly, the phase delay can be calculated according to equation (9) and (10) and compensated.

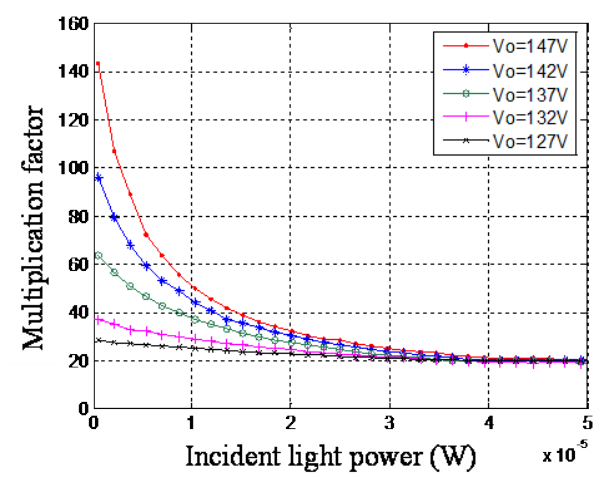

Fig.4. Relationship of incident light intensity and at different bias voltages

\section{Control system design by adjusting bias voltage}

\subsection{SNR of system and optimal $M$ factor}

From section 2, we know that lower the bias voltage to keep the $\mathrm{M}$ factor be a constant can reduce phase delay. But it is a conflict between $\mathrm{M}$ factor and high SNR of the receive system. The relationship between $\mathrm{M}$ factor and the SNR is discussed in order to establish the bias voltage control system. The SNR of current can be calculated by the following equation:

$$
\begin{aligned}
& S N R=\frac{i_{\text {photo }}}{\bar{i}_{n}}=\frac{M \cdot I_{p h}}{\left\{{\overline{i_{T n}}}^{2}+2 e B \cdot\left[I_{D S}+\left(I_{D B}+I_{p h}\right) \cdot M^{2} \cdot F(M)\right]\right\}^{\frac{1}{2}}} \\
& \left\{\begin{array}{l}
{\overline{i_{n}}}^{2}={\overline{i_{T n}}}^{2}+{\overline{i_{D B}}}^{2}+{\overline{i_{D S}}}^{2}+{\overline{i_{P n}}}^{2}=4 K T B / R_{L} \\
{\overline{i_{D B}}}^{2}=2 e M^{2} \cdot F(M) \cdot B \cdot I_{D B} \\
{\overline{i_{D S}}}^{2}=2 e \cdot I_{D S} \cdot B \\
{\overline{i_{P n}}}^{2}=2 e \cdot S(\lambda) \cdot P_{r} \cdot M^{2} \cdot F(M) \cdot B
\end{array}\right.
\end{aligned}
$$

Where $k$ is Boltzmann constant, T is temperature of detector's load resistance $R_{l}$, B is the bandwidth of the receive system, e is electron charge of $1.602 \times 10^{-19} \mathrm{C}, \mathrm{M}$ is multiplication factor. $P_{r}$ is incident light intensity, $F(M)$ is APD excess noise factor, and for reach through structure of silicon APD, $F(M)$ is given by:

$$
F=k M+\left(2-\frac{1}{M}\right)(1-k)
$$

The $\mathrm{M}$ factor and output SNR of APD is plotted in Fig. 5.

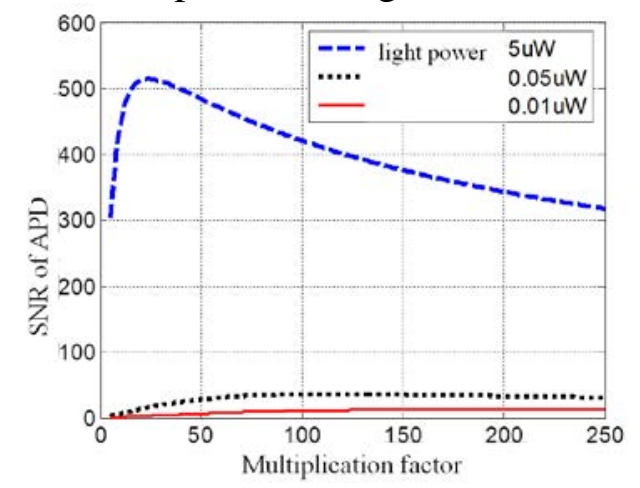

Fig. 3. Curve of multiplication and output SNR of APD 


\subsection{Reducing phase delay by bias voltage adjusting and software compensating}

The SNR is affected by $M$ factor when the incident light is intense. When the incident light intensity is weak, the optimal $\mathrm{M}$ factor is relatively constant. So we establish the control strategy as follows: adjusting the bias voltage to make the APD reaching the optimal SNR when the incident light is intense. When the incident light intensity is weak, we adjust the bias voltage to keep the $\mathrm{M}$ factor at a constant value, the phase delay can be compensated by the optimal $\mathrm{M}$ factor according to equation (10). The control system is presented in Fig. 6. The bias voltage can be controlled by DSP and a DAC. The adjusting procedure is shown in Fig. 7.

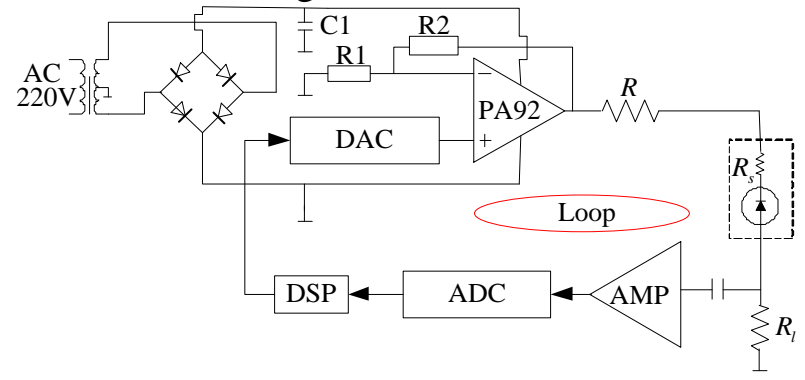

Fig. 4 Multiplication factor control system

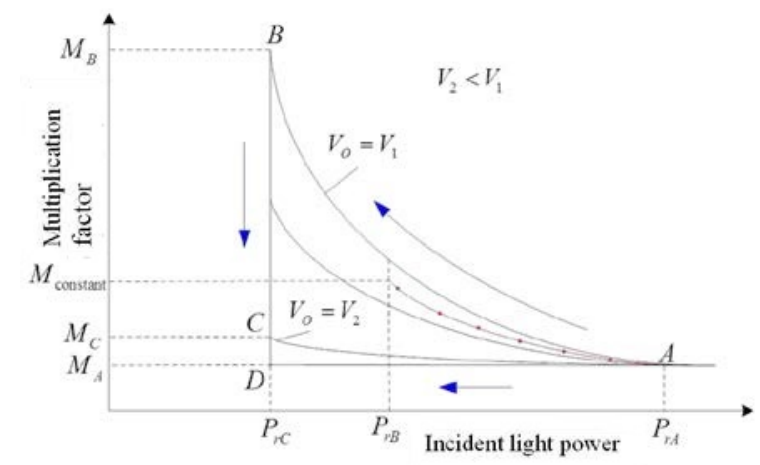

Fig. 5 Procedure of reduce the phase error by adjusting the bias voltage

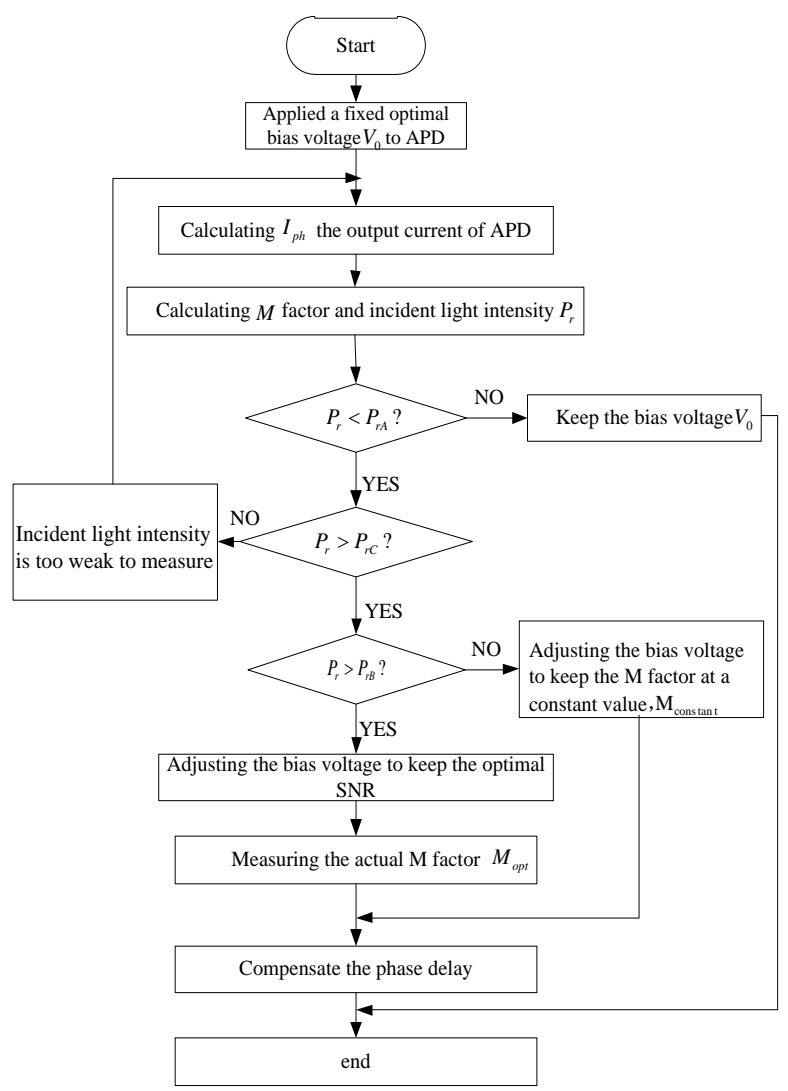

Fig. 6. Flowchart of reducing phase delay by multiplication control and software compensating 
Two curves $\mathrm{AB}$ and $\mathrm{AC}$ belong to the different bias voltage $V_{1}$ and $V_{2}, V_{2}<V_{1}$. when incident light intensity decrease from $\mathrm{A}$ point to $\mathrm{D}$ point at bias voltage $V_{1}$, the $\mathrm{M}$ factor increases from $M_{A}$ to $M_{B}$ along the curve $\mathrm{AB}$. If we control the bias voltage reduce from $V_{1}$ to $V_{2}$, the $\mathrm{M}$ factor jumps from $M_{B}$ down to $M_{C}$, the phase delay is reduced. In order to reduce the phase delay and keep the optimal SNR of the system at the same time, we work out control scheme according to the incident light intensity $P_{r}$. The flowchart of our control system is shown in Fig. 8.

\section{Experimental results}

We change the incident light on APD1 by ATT according to Fig. 2 in $0.5 \mu \mathrm{W} \sim 50 \mu \mathrm{W}$ at modulation frequency of $75 \mathrm{MHz}$ and $7.5 \mathrm{MHz}$. The phase delay is measured after bias adjusting and software compensating. The experimental results are shown in Table 1 .experimental results show that the phase delay caused by APD nonlinearity is less than the $0.04^{\circ}$.

\begin{tabular}{l} 
Table 1 Compensation results of phase delay caused by nonlinear of APD at different modulation \\
\cline { 2 - 4 } \begin{tabular}{lllc} 
Modulation frequency & $f=75 \mathrm{MHz}$ & \multicolumn{2}{l}{ Modulation frequency $f=7.5 \mathrm{MHz}$} \\
$\mathrm{P}_{\mathrm{r}}(\mu \mathrm{W})$ & Phase delay(Degree) & $\mathrm{P}_{\mathrm{r}}(\mu \mathrm{W})$ & Phase delay(Degree) \\
\hline 0.50 & 0.0293 & 0.52 & 0.0245 \\
0.81 & 0.0301 & 0.84 & 0.0287 \\
1.08 & 0.0214 & 1.35 & 0.0154 \\
4.09 & 0.0300 & 4.49 & 0.0134 \\
8.42 & 0.0252 & 8.94 & 0.0291 \\
13.06 & 0.0245 & 13.72 & 0.0223 \\
17.03 & 0.0223 & 17.82 & 0.0175 \\
25.32 & 0.0187 & 25.23 & 0.0226 \\
33.92 & 0.0121 & 33.96 & 0.0151 \\
41.84 & 0.0000 & 41.01 & 0.0000 \\
\hline
\end{tabular}
\end{tabular}

\section{Conclusion}

We have developed an active bias voltage control system combine with software compensation method that can not only reduce the phase delay caused by the nonlinearity of APD but also keep the receive system keep its optimal SNR. The nonlinearity of APD is presented, the relationship among phase delay, multiplication factor, and the incident light intensity are analyzed. The experimental results show that our control system is feasible, flexible, and easier to implement.

\section{References}

[1]. Ichiro Fujima, Shigei Ieasaki, Katuo Seta. High resolution distance meter using optical intensity modulation at 28GHz, Meas. Sci. Techinol., 1998, 9:1049-1052

[2]. A.Ghose, B.Bunz, J.Weide, G, Kompa. Extraction of nonlinear parameters of dispersive avalanche photodiode using pulsed RF measurement and quasi-DC optical excitation. IEEE Trans. Microwave Theory and technique, 2005, 53(6):2082-2087

[3]. Haapalinna, A., Kübarsepp,T., Kärha,P., Ikonen., E.. Measurement of absolute linearity of photodetectors with a diode laser. Meas. Sci, Technol. 1999,Vol.10:1075-1078

[4]. Tsutomu Araki. Optical distance meter using a short pulse width laser diode and a fast avalanche photodiode.Rev. Sci. Instrum.. 1995,66(1):43-47

[5]. Shuko Yokoyama, Norihito Suzuki, Tsutomu Araki. Correction of intensity-dependent phase delay in avalanche photodiodes for high-frequency modulation light. Review. Sci. Instrum. . 1995,66(8):4391-4392 
[6]. Shuko Yokoyama, Akito Okamoto, Tsutomu Araki, et al.Examination to eliminate undesirable phase delay of an avalanche photodiode(APD) for intensity-modulatedlight. Rev.Sci.Instrum., 1995, 66(11):5331-5336

[7]. Stolze A., Kompa,G.. Nonlinear modeling of dispersive photodiodes based on frequency and time measurements. Proc. 26th EuMC, 9-12 September, Prague, Czech Repulblic, 1996,:379-382

[8]. A.Ghose, B.Bunz, J.Weide, G. Kompa. Large signal modeling of avalanche photodiode for correction of optical power dependent nonlinearity, Proc. 34th European Microwave conference, Paris, France, 3-7 October, 2005:357-360

[9]. Abhijit Ghose. Pulsed measurement based nonlinear characterization of avalanche photodiode for the time error correction of $3 D$ pulsed laser radar. Ph.D Dissertation, Electrical engineering/computer science of University of Kassel. 2005

[10]. Atte Haapalinna, Toomas Kübarsepp, Petri Kärhä,et al.. Measurement of the absolute linearity of photodetectors with a diode laser. Mea.Sci.Technol. , 1999, 10:1075-1078 